\title{
Multisensor Input for CPG-Based Sensory-Motor Coordination
}

\author{
R. Héliot and B. Espiau
}

\begin{abstract}
This paper describes a method for providing in real time a reliable synchronization signal for cyclical motions such as steady-state walking. The approach consists in estimating online a phase variable on the basis of several implicit central pattern generator associated with a set of sensors. These sensors can be of any kind, provided their output strongly reflects the timed motion of a link. They can be, for example, spatial position or orientation sensors, or foot sole pressure sensors. The principle of the method is to use their outputs as inputs to nonlinear observers of modified Van der Pol oscillators that provide us with several independent estimations of the overall phase of the system. These estimations are then combined within a dynamical filter constituted of a Hopf oscillator. The resulting phase is a reliable indexing of the cyclic behavior of the system, which can finally be used as input to low-level controllers of a robot. Some results illustrate the efficiency of the approach, which can be used to control robots.
\end{abstract}

Index Terms-Central pattern generator (CPG), oscillator, sensors, sensory-motor coordination.

\section{INTRODUCTION}

A classical way of generating cyclic motions for articulated systems is to synthesize a rhythm generator, called central pattern generator (CPG). The CPG concept comes from biology [1], [2]; it is a small neural network, located at the spinal level, able to generate rhythmic commands for the muscles. CPGs receive inputs from higher parts of the central nervous system, and also from peripheral afferents; thus, its functioning results from an interaction between central commands and local reflexes. The implementation of this artificial CPG is usually achieved either by designing and training an adequate artificial neural network [3], or by using explicitly nonlinear differential equations acting as an adaptable dynamical oscillator [4]. This concept allows us to easily achieve coordination between different limbs, using independent oscillators for each limb, that are coupled together. In that case, phase oscillators are often used in order to easily model the interaction between two oscillators [5], [6].

When several actuators that need to be synchronized are considered, e.g., for multilegged robots, multiple or coupled oscillators can be used, generally under the control of a master CPG. For this kind of classical CPG-based approach, the literature is quite extensive [4], [7]-[9]. However, the need for adaptation of the system to environmental changes, external requirements, or proprioceptive information through sensory signals is more rarely addressed. We can nevertheless refer the reader to a few recent papers in the field that give a good idea of the state of the art; in [10], a robot fish can avoid obstacles through infrared sensors, the output of that will allow the system to select CPG model strategies among a set of predefined ones. Fukuoka et al. [11] modulate the phase of a neural oscillator on the basis of the

Manuscript received January 31, 2007; revised October 11, 2007. This paper was recommended for publication by Associate Editor A. Takanisi and Editor F. Park upon evaluation of the reviewers' comments.

R. Héliot is with the Commissariat Energie Atomique-Laboratoire d'Electronique et de Technologie de l'Information (CEA-LETI), 38000 Grenoble Cedex, France. He is also with the Institut National de Recherche en Informatique et en Automatique (INRIA) Rhône Alpes, 38334 Saint Ismier Cedex, France (e-mail: rodolphe.heliot@inrialpes.fr).

B. Espiau is with the Institut National de Recherche en Informatique et en Automatique (INRIA) Rhône Alpes, 38334 Saint Ismier Cedex, France (e-mail: bernard.espiau@inria.fr).

Digital Object Identifier 10.1109/TRO.2008.915433 


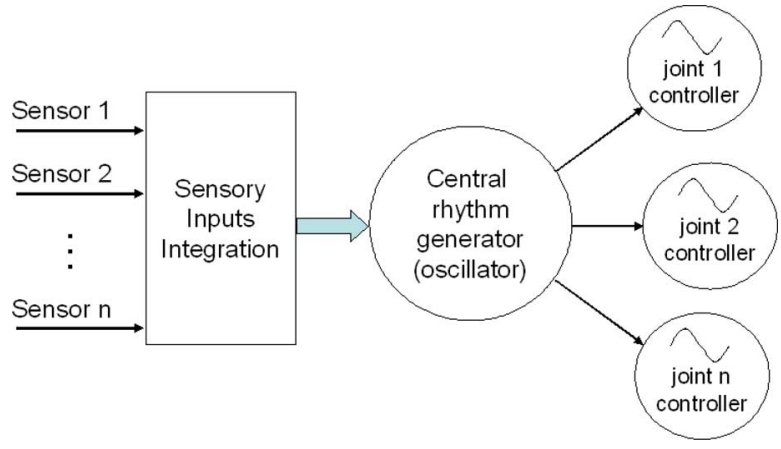

Fig. 1. CPG-based architecture with sensory inputs integration.

measurement of body angle for driving a quadruped robot on rough terrain. A similar approach is reported in [12]. In [13], the synchronization of the walk of a biped robot is ensured by triggering an oscillator upon the event emitted by a touch sensor placed on the foot. Simoni and DeWeerth [14] propose a control scheme for a single-link rigid system based on a silicon neural CPG, which integrates an internal feedback from a position encoder.

Nevertheless, there remains a lack in design tools in the domain of oscillators and synchronization. A lot of analysis tools are available [15], [16], but few synthesis ones, as pointed out by Bailey [17] and Righetti et al. [18]. In fact, it appears that the question of using continuously a set of sensor measurements as driving inputs to an artificial CPG aimed at controlling several links in a safe way is still an open question. In this paper, we propose to address this question in the following way: we consider a set of nonlinear oscillators, each of them is forced by the output of a sensor that gives a time-indexed first-order information on the motion. Whatever is the used technology, this information is assumed to represent continuously the cyclic motion of the system. We then reconstruct the useful part of the state of every oscillator through adequate observers and combine them to finally estimate a single relevant value of the whole synchronized motion: its phase. It should be emphasized that this phase estimation has to be done online when it has to be used in a control scheme; therefore, classical offline phase estimation methods such as Hilbert transform [19] cannot be used.

Finally, our goal is to integrate a set of inputs within a robot control architecture based on a master-oscillator-based CPG driving the different joints (see Fig. 1). Although such a controller is not a pure reflection of the biology, this simplified architecture is very adequate to control a robot where the coordination of many degrees of freedom is a critical issue, as it is the case in bipedal robotics. Here, every individual link motion synchronized with respect to the inputs can be generated using the previously estimated phase as a time-scaling variable, and fed into low-level controllers.

The paper is organized as follows: after having introduced some basic concepts, we describe the methods used for designing an oscillator, its related observer, and the phase estimation. We then present the method of fusion of individually estimated phases, and finally, give some results from real experimental data. Future workplans are drawn in Section IV.

\section{METHODS}

The basic assumption in the proposed approach is that considered motions reflect a cyclical or a periodic activity: this means that all involved signals (sensor outputs, control variables, etc.) can be described along a cycle. In order to assign to these variables a kind of relative position on the cycle, the phase $\varphi$ can be introduced as a coordinate along the limit cycle [15], i.e., as a variable that grows uniformly in the

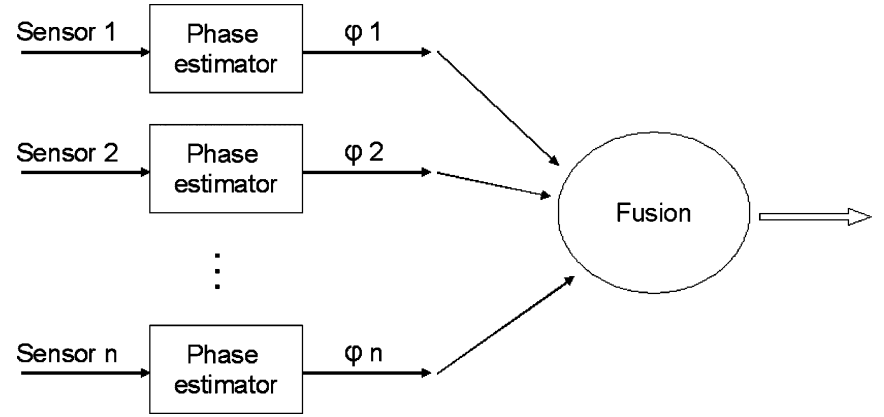

Fig. 2. Principle of the integration of sensor signals.

direction of the motion and gains $2 \pi$ during each rotation, thus obeying the equation

$$
\frac{d \varphi}{d t}=\omega_{0}
$$

where $\omega_{0}=2 \pi / T_{0}$ is the frequency of the oscillations when unforced.

Knowing that several variables have to be combined in order to find a unique synchronization signal, the proposed method works in two steps (see Fig. 2):

1) phase estimation for each sensor;

2) fusion of individual phases in a global phase variable.

In the following, we will take a bipedal gait observation as an example, with three different sensors: a thigh inclination sensor, a shank inclination sensor, and pressure insoles on both feet. When needed, especially in the phase estimation part (Section II-A), we will focus on a specific sensor, the thigh inclination angle signal, in order to illustrate the method.

\section{A. Phase Estimation}

A phase estimator can be issued from an oscillator that has to synchronize with a given cyclic sensory input. The methodology we use to synthesize such an oscillator can be summarized in the following steps.

1) Build a phenomenological model of the evolution of the sensor measurements resulting from a cyclical motion, under the form of a nonlinear oscillator.

2) Build an observer of this system, wherein the injected actual sensor measurements are present.

3) Compute the phase from the estimated state variables.

1) Nonlinear Oscillator Model: Let us now consider the modeling under the form of an oscillator of the time evolution of the thigh inclination in standard human gait. From a mechanical point of view, the motion of a human body restricted to a tree-form kinematical structure with variable unilateral ground contacts can be modeled using a Newton-Euler or a Lagrangian approach, leading to a nonlinear second-order system; from a biomechanical point of view, the standard steady-state human walking corresponds to minimal metabolic energy consumption and is naturally reached after some transient steps. All these facts reflect at the link level, therefore in the thigh angle itself and its measurement. It finally appears that searching for an oscillator of second-order type and exhibiting a limit cycle is a natural way of modeling the steady-state behavior of a human leg link measured with an adequate sensor.

As detailed in [20], we have, therefore, chosen a Van der Pol equation as a model of the system, the damping term of that has been modified to take into account asymmetrical patterns:

$$
\ddot{x}-\mu\left(1-b x-x^{2}\right) \dot{x}+\omega_{0}^{2} x=0 .
$$




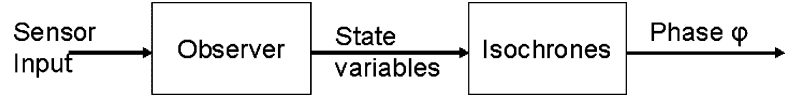

Fig. 3. Computation flow of the phase estimator.

2) Observer Design: After having computed offline the three parameters of the oscillator that give the best fitting of its limit cycle with respect to a set of recorded actual measurements, a dedicated nonlinear observer can be built under the form

$$
\Sigma^{\prime}:\left\{\begin{array}{l}
\dot{z}=-z+\left(k_{1}-\omega_{0}^{2}\right) y+k_{2} y^{2}+k_{3} y^{3} \\
\hat{x}_{1}=y \\
\hat{x}_{2}=z-k_{1} y-k_{2} y^{2}-k_{3} y^{3}
\end{array}\right.
$$

where $k_{i}$ is the coefficient dependent on the oscillator parameters and $y$ is the current sensor output. All technical developments can be found in [20].

3) Phase Estimation Through Isochrones: By injecting the measurement $y$ as an input to the observer, we get an estimation of the state variables $\hat{x}_{1}$ and $\hat{x}_{2}$ introduced in (3). Since this observer is itself an oscillator, its phase can be computed from its state variables, even if the estimated state does not belong exactly to the limit cycle. This can be done using isochrones: the limit cycle of an oscillator can be parametrized using a phase variable, which can be extended in its neighborhood. The so-called isochrones sets can be defined in the vicinity of the limit cycle, demanding that the phase be constant on each isochrone. We thus get a mapping from the phase space to the phase variable: $\varphi=I(\mathbf{x})$.

4) Summary: Finally, the online computation scheme for phase estimation is the following (see Fig. 3).

1) Inject the sensor measurement $y_{k}$ in the adapted observer, to compute its state variables $\hat{x}_{k}$.

2) From the observer state variables $\hat{x}_{k}$, compute the phase $\tilde{\varphi}_{k}$ of the oscillator through isochrones.

An important remark has to be made here: the strong synchronization of the observer with the given input signal is ensured with this method; thanks to observer theory, it can be assessed that the behavior of the observer will asymptotically match the behavior of the observed system. In practice, the convergence time of the observer is extremely small with respect to the period of the motion, which means that the performed filtering is phase-shift free.

\section{B. Fusion of the Estimated Phases}

After having estimated different phases $\tilde{\varphi}_{k}(t)$ in Section II-A, we now address the issue of phases fusion along two possible lines.

1) A Stochastic Filter Approach: We have seen that the phase $\varphi$ was a way of indexing the cycle and its neighborhood with respect to the time. An ideal model of the phase is, therefore,

$$
\frac{d \varphi}{d t}=\omega_{0}
$$

To estimate a global phase of the system, we have to use the individual estimates $\tilde{\varphi}_{k}(t), k=1, \ldots, N$ provided by the $N$ independent observers driven by the sensor inputs. These estimates can, therefore, be considered as noisy measurements of the phase $\tilde{\varphi}_{k}(t)=\varphi(t)+\epsilon_{k}(t)$, the dynamics of which is described by (4). We can now set as a state variable

$$
X=\left(\begin{array}{c}
x_{1} \\
x_{2}
\end{array}\right)=\left(\begin{array}{c}
\varphi \\
\omega_{0}
\end{array}\right)
$$

and finally, write the linear model as

$$
\left\{\begin{array}{l}
\dot{X}(t)=A X(t)+E_{1}(t) \\
Y(t)=C X(t)+E_{2}(t)
\end{array}\right.
$$

where

and where

$$
A=\left[\begin{array}{ll}
0 & 1 \\
0 & 0
\end{array}\right]
$$

$$
E_{1}(t)=\left[\begin{array}{c}
0 \\
e_{1}(t)
\end{array}\right]
$$

is a state perturbation allowing to cope with phase model errors. Also, we obtain

$$
\begin{gathered}
Y^{t}=\left[\tilde{\varphi}_{1} \cdots \tilde{\varphi}_{k} \cdots \tilde{\varphi}_{N}\right]^{T} \\
C=\left[\begin{array}{cc}
1 & 0 \\
\vdots & \\
1 & 0
\end{array}\right] \quad \text { and } \quad E_{2}=\left[\begin{array}{l}
\epsilon_{1}(t) \\
\vdots \\
\epsilon_{N}(t)
\end{array}\right]
\end{gathered}
$$

where $\epsilon_{k}(t)$ is a Gaussian white noise.

The associated continuous Kalman filter can be written as

$$
\hat{\dot{X}}(t)=A \hat{X}(t)+K(t)(Y(t)-C \hat{X}(t))
$$

with $\hat{X}(0)=\left(\begin{array}{c}\hat{\varphi}\left(t_{0}\right) \\ \omega_{0}\end{array}\right)$.

$K(t)$ is the solution of a classical Riccatti equation involving the covariance matrices $\Sigma_{1}$ and $\Sigma_{2}$ of $E_{1}$ and $E_{2}$, respectively, and finally,

$$
\hat{\varphi}(t)=\hat{x_{1}}(t)(\bmod 2 \pi) \text {. }
$$

This method requires the estimation of $\Sigma_{1}$ and $\Sigma_{2}$. For $\Sigma_{2}$, this can be done using a training stage dedicated to parameter identification. Due to the particular form of (4), a single linear regression on each time-indexed records of $\tilde{\varphi}_{k}$ along a cycle gives an indication of the confidence. Concerning $\Sigma_{1}$, the adjustment has to be done with respect to the capacity of tracking period variations that is desired.

It should be noticed that the behavior of this Kalman filter is optimal when noises are white and Gaussian. It is clear that it is not the case. Furthermore, the ability of the filter to track with a delay, small enough, the evolution of the system is linked to the realism of the models, which is not always easily ensured. That is why we have preferred to use, again, a nonlinear oscillator, which acts in some sense as an adapted dynamical filter.

2) A Dynamical System Approach: Another possibility is to filter the different phase measurements through a dynamical system. In the CPG framework, a central oscillator often plays the role of "pacemaker" (see Fig. 1). An idea is then to force this central oscillator with the phase estimations $\tilde{\varphi}_{k}$, so that it synchronizes with them. Oscillator forcing for synchronization has already been studied [15] and applied successfully in a CPG-based robot control application [18].

The Hopf oscillator is a good candidate for playing this central role, since it has a quasiharmonic limit cycle. It is described as

$$
\begin{aligned}
& r=\sqrt{x^{2}+y^{2}} \\
& \dot{x}=\gamma\left(\mu-r^{2}\right) x-\omega y \\
& \dot{y}=\gamma\left(\mu-r^{2}\right) y+\omega x
\end{aligned}
$$

with $\gamma, \mu$, and $\omega>0$, and $\mu$ is the radius of the limit cycle (which is almost a perfect circle), $\gamma$ is a damping coefficient, directly linked to the convergence speed toward the limit cycle, and $\omega$ defines the period of oscillations.

By adding a forcing term to (8), we obtain

$$
\begin{aligned}
& r=\sqrt{x^{2}+y^{2}} \\
& \dot{x}=\gamma\left(\mu-r^{2}\right) x-\omega y+C_{\text {forc }} \\
& \dot{y}=\gamma\left(\mu-r^{2}\right) y+\omega x+S_{\text {forc }}
\end{aligned}
$$




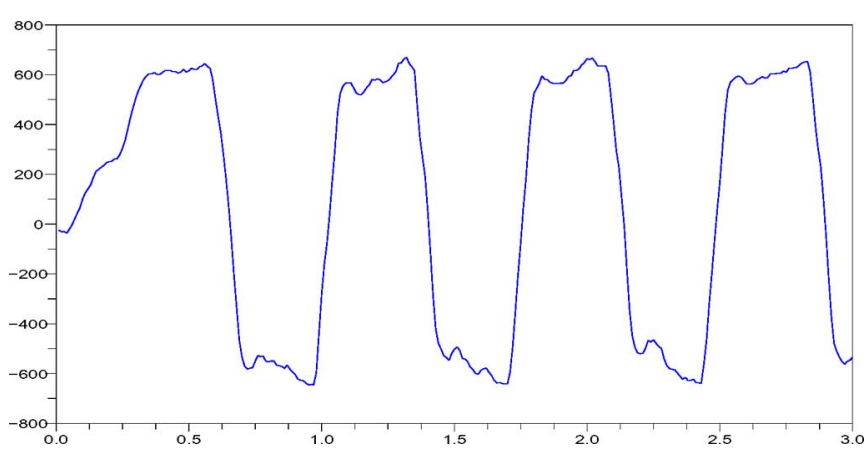

Fig. 4. Composite pressure signal built from pressure insoles measurements.

where

$$
\begin{aligned}
C_{\text {forc }} & =\sum_{k=1}^{n} c_{k} \cos \left(\tilde{\varphi}_{k}\right) \\
S_{\text {forc }} & =\sum_{k=1}^{n} c_{k} \sin \left(\tilde{\varphi}_{k}\right)
\end{aligned}
$$

represent the forcing terms, with $\sum_{n}^{k=1} c_{k}=1$. Coefficients $c_{k}$ can be seen as confidence coefficients in each phase variable $\tilde{\varphi}_{k}$, and can be directly derived from the statistical analysis presented in Section II-B1. The final phase $\hat{\varphi}$ is the phase of the Hopf oscillator and can be easily extracted using polar coordinates:

$$
\hat{\varphi}=\arctan \frac{y}{x}
$$

The main advantage of this method is that we benefit from a smoothing effect due to the dynamical model. Noise and transient values are naturally filtered.

\section{IMPLEMENTATION AND RESULTS}

\section{A. Sensors}

1) Angle Estimation: The inclination of the thigh is measured by an attitude sensor, developed by the Commissariat Energie AtomiqueLaboratoire d'Electronique et de Technologie de l'Information (CEA-LETI), which associates three microaccelerometers and three micromagnetometers in a small volume. Owing to an adequate processing, this sensor provides with an estimation of the orientation in space of the segment to which it is attached [21]. Fig. 5 (top) shows an example of the obtained estimation of thigh inclination during human gait. The same sensing procedure was used for shank inclination reconstruction.

2) Pressure Insoles: We also used feet pressure information as a sensory input. To this end, a Pedar [22] system was employed: it is an accurate measuring system of the pressure distribution that represents local loads between the foot and the shoe. To obtain a single sensory signal from data from both the feet, we build the composite pressure signal as

$$
P_{c}=\sum_{i} p_{\text {right foot }}^{i}-\sum_{i} p_{\text {left foot }}^{i}
$$

where the $p^{i}$ s are local load measurements for each foot. In such a way, $P_{c}$ is positive when the right foot stands on the ground and left leg is in swing phase and negative in the opposite case. Fig. 4 shows a typical plot of this pressure signal.

\section{B. Phase Estimation Results}

As indicated earlier, we recorded measurements during human gait from three different sensors: a thigh inclination, a shank inclination,
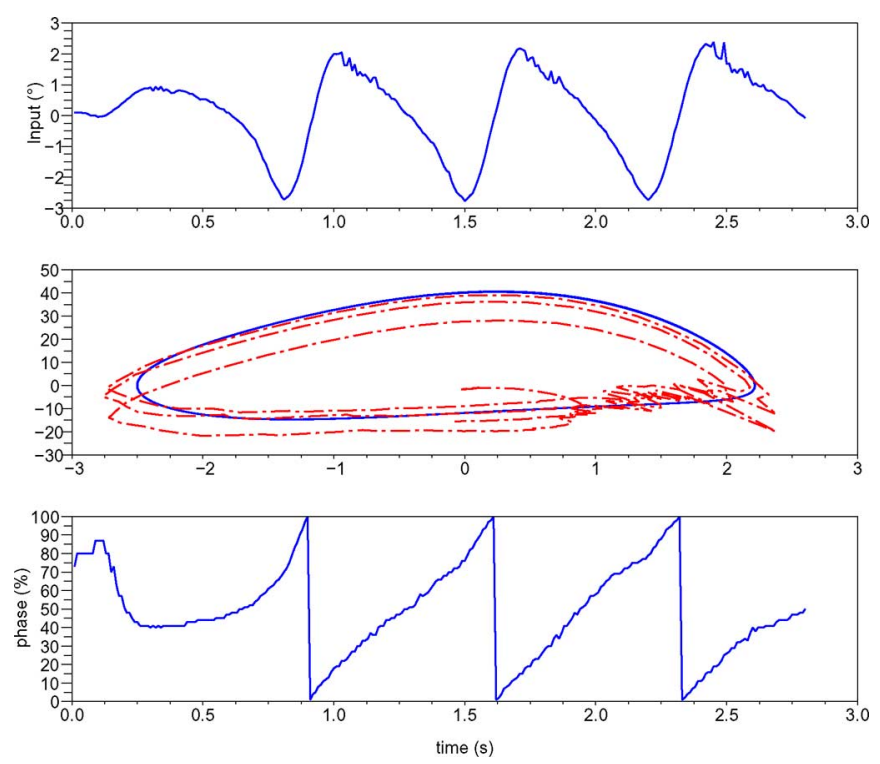

Fig. 5. Estimation of one-sensor phase. Sensor input (thigh inclination) (top). Estimated state variables of the observer in the phase space (dotted line), compared to the reference limit cycle provided by the oscillator model (middle). Estimated phase (from $0 \%$ to $100 \%$ ) (bottom).

and insoles pressure. As an illustration of the method, we present here phase estimation results for a single sensor only: the thigh inclination. Fig. 5 shows three consecutive measurement cycles, together with the reference limit cycle of the Van der Pol oscillator; it should be noticed that, although the estimated state variables do not always belong to the limit cycle, they stay rather close to it.

Using the isochrones-based estimation of the phase from the reconstructed states, we get a phase estimation that is monotonous, quasipiecewise linear, and synchronous to the input signal. Nevertheless, noises and transient biases are still present. That is why it is of great interest to combine several phase information, in order to get a more reliable result.

\section{Fusion Results}

Fig. 6 presents the result of the fusion of individual estimated phases using the dynamical system approach (Section II-B2). We can clearly notice that the three estimated phases $\tilde{\varphi}_{k}$ are all noisy, and some of them with large biases. The final estimated phase $\hat{\varphi}$ is nicely filtered: all noise alterations have disappeared, the signal is perfectly smooth and almost linear.

Remark: One could wonder why the sensor inputs are not directly injected into a Hopf oscillator. In fact, since the Hopf oscillator has a quasi-harmonic behavior, only inputs with sinusoidal shapes would lead to a correct phase estimation. In some sense, the "phase estimation" stage provides with a conditioned input, i.e., a linearly growing phase, without distortions. Besides, as said earlier, it ensures the synchronization properties of the system.

\section{Bipedal Robot Teleoperation}

This experiment consisted in installing three sensors on the leg and feet of a human, in order to compute online a biped robot command, such that the robot "follows" the rhythm of the human gait. This was done as follows: basically, the desired trajectories of each active joint of the robot (ankle, knee, and hip sagittal angles on both legs of the biped robot) are standard Winter's patterns [23], and are tracked using 

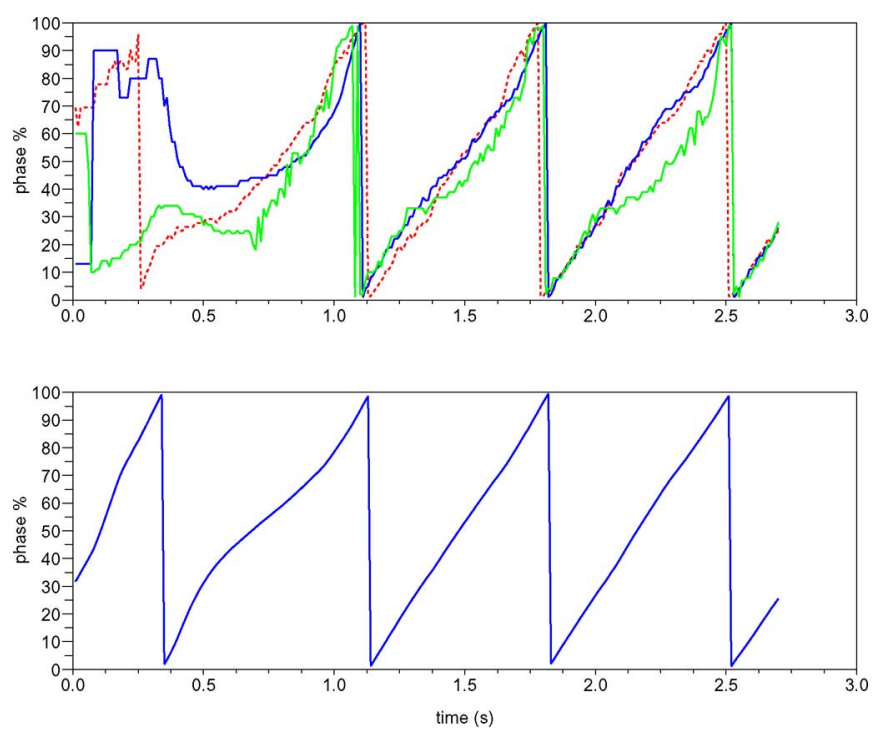

Fig. 6. Results of phases fusion. Estimated phases from three different sensors (Top). Final estimated phase (bottom).
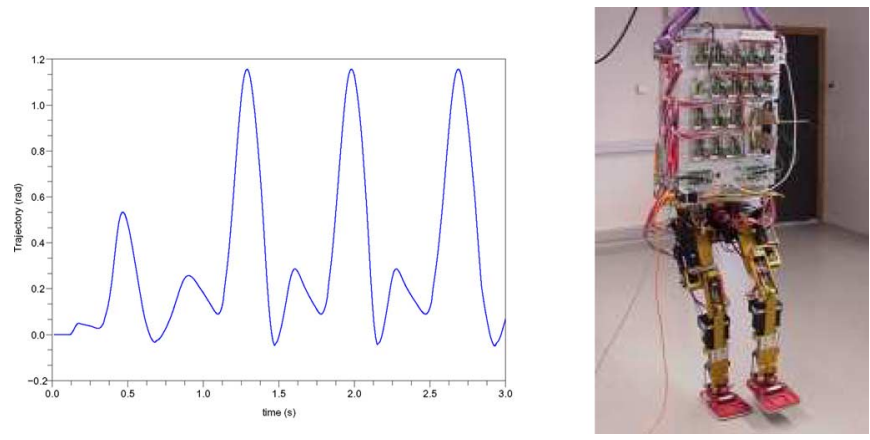

Fig. 7. Generated trajectory for one DOF (knee angle) of the biped robot (on the right).

a PID controller with gravity and friction compensation. The phase estimated by the method presented earlier allows them a kind of online time scaling of these trajectories, realizing in that way the aimed synchronization. The experiments were conducted on the BIP robot, the robot being hanged. This 15-jointed robot [24] has two legs and a trunk, the legs sizes and masses distribution corresponding to the ones of a standard male adult. We thus fully validated the desired sensory-motor coordination, following the computation scheme presented in Fig. 1. An example of the trajectory generated for a single depth of field (DOF) is shown in Fig. 7.

\section{DISCUSSION}

In this paper, we have presented a new CPG-type method for online phase estimation using dynamical systems. Sensor signals are used to drive a set of modified Van der Pol oscillators, which provide through an adequate observer several independent estimations of the overall phase of the system. These estimations are again combined within a dynamical filter consisting of a Hopf oscillator. The resulting phase is a reliable indexing of the cyclic behavior of the system. A major feature of the approach is the strong synchronization of the whole system with respect to the perceived cyclic motion. Potential applications of the method are numerous: in robotics, it can be used for teleoperating a biped robot synchronized with external signals, such as a real human walk. From another point of view, the method allows to strongly generate synchronized trajectories for four- or six-legged robots. The method could be extended to incorporate internal sensor feedback in complement of the external measurements presently used. In that case, note that the stability of the closed-loop system should be assessed.

\section{REFERENCES}

[1] S. Grillner, "Neurobiological bases of rythmic motor acts in vertebrates," Science, vol. 228, pp. 143-149, 1985.

[2] A. Cohen, S. Rossignol, and S. Grillner, Neural Control of Rhyhtmic Movements in Vertebrates. New York: Wiley, 1988.

[3] K. Matsuoka, "Sustained oscillations generated by mutually inhibiting neurons with adaptation," Biol. Cybern., vol. 52, no. 6, pp. 367-376, 1985.

[4] L. Righetti and A. J. Ijspeert, "Programmable central pattern generators: An application to biped locomotion control," in Proc. 2006 IEEE Int. Conf. Robot. Autom., May 15-19, pp. 1585-1590.

[5] A. Cohen, P. Holmes, and R. Rand, "The nature of the coupling between segmental oscillators of the lamprey spinal generator for locomotion: A mathematical model," J. Math. Biol., vol. 13, pp. 345-369, 1982.

[6] N. Kopell and G. Ermentrout, "Symmetry and phaselocking in chains of weakly coupled oscillators," Commun. Pure Appl. Math., vol. 39, pp. 623-660, 1986.

[7] M. M. Williamson, "Neural control of rhythmic arm movements," Neural Netw., vol. 11, no. 7/8, pp. 1379-1394, 1998.

[8] G. Taga, "Nonlinear dynamics of the human motor control," presented at the Adapt. Motion Anim. Mach. (AMAM 2000), Brighton, U.K.

[9] A. Ijspeert, "A connectionist central pattern generator for the aquatic and terrestrial gaits of a simulated salamander," Biol. Cybern., vol. 84, no. 5, pp. 331-348, 2001.

[10] L. Wang, S. Wang, Z. Cao, M. Tan, C. Zhou, H. Sang, and Z. Shen, "Motion control of a robot fish based on CPG," in Proc. IEEE Int. Conf. Ind. Technol. (ICIT), 2005, pp. 1263-1268.

[11] Y. Fukuoka, T. Mimura, N. Yasuda, and H. Kimura, "Integration of multi sensors for adaptive walking of a quadruped robot," in Proc. IEEE Int. Conf. Multisensor Fusion Integr. Intell. Syst., Aug. 2003, pp. 21-26.

[12] H. Dong, M. Zhao, J. Zhang, and N. Zhang, "CPG-based adaptive dynamic control of a quadruped robot with sensory feedback," in Proc. 9th Int. Conf. Climbing Walking Robots, 2006, pp. 77-81.

[13] S. Aoi and K. Tsuchiya, "Stability analysis of a simple walking model driven by a nonlinear oscillator," in Proc. IEEE Int. Conf. Syst., Man, Cybern., 2004, vol. 5, pp. 4450-4455.

[14] M. F. Simoni and S. DeWeerth, "Sensory feedback in a half-center oscillator model," IEEE Trans. Biomed. Eng., vol. 54, no. 2, pp. 193-204, Feb. 2007.

[15] A. Pikovsky, R. Rosenblumn, and J. Kurths, Synchronization, A Universal Concept in Nonlinear Sciences. Cambridge, U.K.: Cambridge Univ. Press, 2001.

[16] J. Guckenheimer and P. Holmes, Nonlinear Oscillations, Dynamical Systems and Bifurcations of Vector Fields. New York: Springer-Verlag, 1990.

[17] S. Bailey, "Biomimetic control with a feedback coupled nonlinear oscillator: Insect experiments, design tools, and hexapedal robot adaptation results," Ph.D. dissertation, Stanford Univ., Stanford, CA, Jul. 2004.

[18] L. Righetti, J. Buchli, and A. Ijspeert. (2006), "Dynamic hebbian learning in adaptive frequency oscillators" Physica D [Online]. 216(2), pp. 269281. Available: http://dx.doi.org/10.1016/j.physd.2006.02.009

[19] R. Bracewell, The Fourier Transform and Its Applications, 2nd ed. New York: McGraw-Hill, 1986.

[20] R. Heliot and B. Espiau, Online generation of cyclic leg trajectories synchronized with sensor measurement, [Online]. Robot. Auton. Syst., Available: DOI: http//dx.doi.org/10.1016/j.robot.2007.09.019.

[21] S. Bonnet and R. Heliot, "A magnetometer-based approach for studying human movements," IEEE Trans. Biomed. Eng., vol. 54, no. 7, pp. 1353 1355, Jul. 2007.

[22] [Online]. Available: http://www.novel.de/productinfo/systems-pedar.htm.

[23] D. Winter, The Biomechanics and Motor Control of Human Gait. Waterloo, ON, Canada: Univ. of Waterloo Press, 1987.

[24] C. Azevedo and the Bip team, "Control architecture and algorithms of the anthropomorphic biped BIP2000," presented at the Int. Symp. Mobile Climbing Walking Robots (CLAWAR), Madrid, Spain, 2000. 